\title{
Regulation of carotenoid synthesis and accumulation in plants*
}

\author{
Francis X. Cunningham, Jr. \\ Department of Cell Biology and Molecular Genetics, University of Maryland, \\ College Park, MD 20742, USA
}

\begin{abstract}
Although genes that encode most enzymes of the carotenoid pathway in higher plants have been identified, the regulatory mechanisms that govern the synthesis and accumulation of carotenoid pigments are still obscure. Recent findings relevant to two aspects of carotenoid pathway control are reviewed: availability of substrate and pathway branching. Experimental approaches that are likely to enhance our understanding of carotenoid pathway regulation are also described.
\end{abstract}

\section{INTRODUCTION}

The amounts and identities of the various carotenoids in the photosynthetic membranes of green plants are relatively well conserved. A handful, typically including lutein, $\beta$-carotene, violaxanthin, neoxanthin, and zeaxanthin, account for most of the carotenoid pigment in the chloroplasts of many plant and algal species [1]. The biosynthesis and accumulation of these carotenoids in developing chloroplasts proceed in concert with the assembly of the light-harvesting antennae and reaction centers with which these pigments are in large part associated [2]. Genetic modifications that reduce or prevent synthesis of one or more of these carotenoids may be compensated by increases in others so that the total carotenoid content in the photosynthetic membranes is not much affected [3-7]. Such observations make clear that robust feedback mechanisms exert control over carotenoid synthesis and accumulation in plant chloroplasts.

Carotenoid pigmentation in non-green plant plastids, in contrast, ranges broadly both in quantity and composition. The total amount of the carotenoids may vary from little or none (as in white flower petals) to quite substantial quantities (as in the dark orange petals of certain marigold varieties). The pigments may include those common in the photosynthetic membranes (e.g., the lutein of marigold flower petals), consist of earlier pathway intermediates (e.g., lycopene in red tomato fruits), or be derived from carotenoids normally found in the chloroplasts (e.g., capsanthin and capsorubin, formed from violaxanthin in red pepper fruits).

What mechanisms are employed by plants to specify and adjust the amounts and identities of the various carotenoids that are accumulated in green and non-green plastids? The answer to this question has many parts, and much remains to be learned. There is abundant evidence to indicate that the reaction catalyzed by phytoene synthase (PSY) is an important control point for regulation of flux into and through the carotenoid pathway [8]. This reaction will not be discussed here. Instead, following a brief update on genes and enzymes of the pathway, I will review what has been learned recently regarding two other likely control points of the carotenoid pathway in plants: the availability of substrate and branching of the pathway.

*Lecture presented at the $13^{\text {th }}$ International Symposium on Carotenoids, Honolulu, Hawaii, USA, 6-11 January 2002. Other presentations are presented in this issue, pp. 1369-1477. 
Genes encoding the enzymes for nearly every step of the carotenoid pathway in green plants have now been identified. The incidence of each pathway gene in the completely sequenced genome of the green plant Arabidopsis thaliana is given in Table 1. With the single exception of the $\beta$-ring hydroxylase (Chy-b gene), only one copy of each carotenoid pathway gene is present in Arabidopsis (the products of the Ipi and Ggps genes are shared with other isoprenoid pathways). It was anticipated that one of the two Chy- $b$ might actually encode an $\varepsilon$-ring hydroxylase. However, both gene products appear to be bona fide $\beta$-ring hydroxylases: carotenoids with $\beta$-rings are good substrates whereas those with $\varepsilon$-rings are poor substrates for each enzyme in a heterologous Escherichia coli system $[9,10]$. It is possible that one (or both) $\mathrm{CHYb}$ also serves as an $\varepsilon$-ring hydroxylase, perhaps requiring another polypeptide for proper orientation and efficient function.

Table 1 Carotenoid pathway genes in Arabidopsis thaliana.

\begin{tabular}{llc}
\hline Gene & \multicolumn{1}{c}{ Enzyme } & Family Members* \\
\hline Ipi & isopentenyl diphosphate isomerase & 2 \\
Ggps & geranylgeranyl diphosphate synthase & 11 \\
Psy & phytoene synthase & 1 \\
Pds & phytoene desaturase & 1 \\
Zds & C-carotene desaturase & 1 \\
CrtISO & carotene isomerase & 1 \\
Ptox & plastid terminal oxidase & 1 \\
Lcy-b & lycopene $\beta$-cyclase & 1 \\
Lcy-e & lycopene -cyclase & 1 \\
Chy-b & B-ring hydroxylase & 2 \\
Chy-e & E-ring hydroxylase & not identified \\
Zep & zeaxanthin epoxidase & 1 \\
Vde & violaxanthin de-epoxidase & 1 \\
Nsy & neoxanthin synthase & no ortholog \\
\hline
\end{tabular}

* Remote homologs (i.e., <30\% identity for deduced amino acid sequences) of Zep, Ptox, crtISO, and a few other genes are not included. Other than for some members of the Ggps family (only 5 of the 11 have so far been examined; see [14]), the appropriate enzymatic activity has been demonstrated for all family members.

Surprisingly, no counterpart of the recently identified neoxanthin synthase gene $(N s y)$ [11,12] is present in the Arabidopsis genome. Polypeptides encoded by Nsy genes are highly similar in amino acid sequence to lycopene $\beta$-cyclases (LCYb), and the tomato $N$ sy gene product has $\beta$-cyclase activity and accounts for the high $\beta$-carotene content of the fruits of the tomato $B$ mutant [13]. It may be that the Arabidopsis $\mathrm{LCYb}$ functions as both $\beta$-cyclase and neoxanthin synthase.

The two genes listed in Table 1 that encode enzymes shared with other isoprenoid pathways, Ipi and Ggps, are present in multiple copies in the Arabidopsis genome. Both Ipi and as many as 6 of the 11 Ggps specify polypeptides with $\mathrm{N}$ terminal extensions that likely target them to the plastid compartment. Because GGPS and IPI have been found to co-purify with PSY (see below), an important question relevant to pathway regulation is whether one of the plastid-targeted GGPS or IPI is dedicated to carotenoid synthesis.

\section{SUPPLY OF SUBSTRATES (IPP AND DMAPP) FOR CAROTENOID BIOSYNTHESIS}

Carotenoids are isoprenoids. The five carbon building blocks that serve as precursors for the synthesis of carotenoids and other isoprenoid compounds, isopentenyl diphosphate (IPP) and dimethylallyl diphosphate (DMAPP), are produced in two different compartments and by two different pathways in 
plant cells (Fig. 1). The well-known mevalonate (MVA) pathway in the cytosol/endoplasmic reticulum begins with acetyl-CoA and proceeds in linear fashion to IPP, which is then reversibly converted to DMAPP in a reaction catalyzed by IPP isomerase (IPI) [15].

The recently recognized methylerythritol (MEP) pathway occurs in plant plastids, in cyanobacteria, and in certain other bacteria [16; see 17 for a recent update on this incompletely elucidated pathway], and utilizes pyruvate and glyceraldehyde-3-phosphate (GAP) as the initial substrates (Fig. 1). In contrast to the MVA pathway, DMAPP and IPP are produced separately via a branching of the MEP pathway [18]. Even so, IPP isomerase, the enzyme that serves as the terminal enzyme of the cytosolic MVA pathway (Fig. 1), is also present in plastids [19,20].

Carotenoids in plants are synthesized in the plastids. Are the IPP and DMAPP utilized for carotenoid synthesis produced solely via the plastid MEP pathway or does the cytosolic MVA pathway also contribute? Does the source of IPP/DMAPP for plastid isoprenoid synthesis depend on the stage of development, the type of tissue, or the type of plastid (e.g., etioplast, chloroplast, chromoplast, or amyloplast)?

Although there are some indications of compartmental "crosstalk", isoprenoid synthesis in both green and non-green plastids of many plants has been found to rely primarily on IPP and DMAPP produced via the MEP pathway. Much of the evidence in support of an MEP pathway origin for plastid isoprenoids comes from analyses of the distribution of label in certain isoprenoid pathway end-products after incubation of plants or algae with ${ }^{13} \mathrm{C}$-labeled glucose or ${ }^{13} \mathrm{C}$-labeled 1-deoxy-D-xylulose (DOX) [reviewed in 21]. The effects of specific MVA and MEP pathway inhibitors and the phenotypic consequences of a mutation in an Arabidopsis gene encoding the MEP pathway enzyme deoxyxylulose-5phosphate synthase (DXS) lend further support. The application of the MEP pathway inhibitor fosmidomycin (an inhibitor of deoxylulose-5-phosphate reductoisomerase, DXR, the first enzyme specific to the MEP pathway; see Fig. 1) reduces lycopene accumulation in tomato fruits [22], whereas the MVA

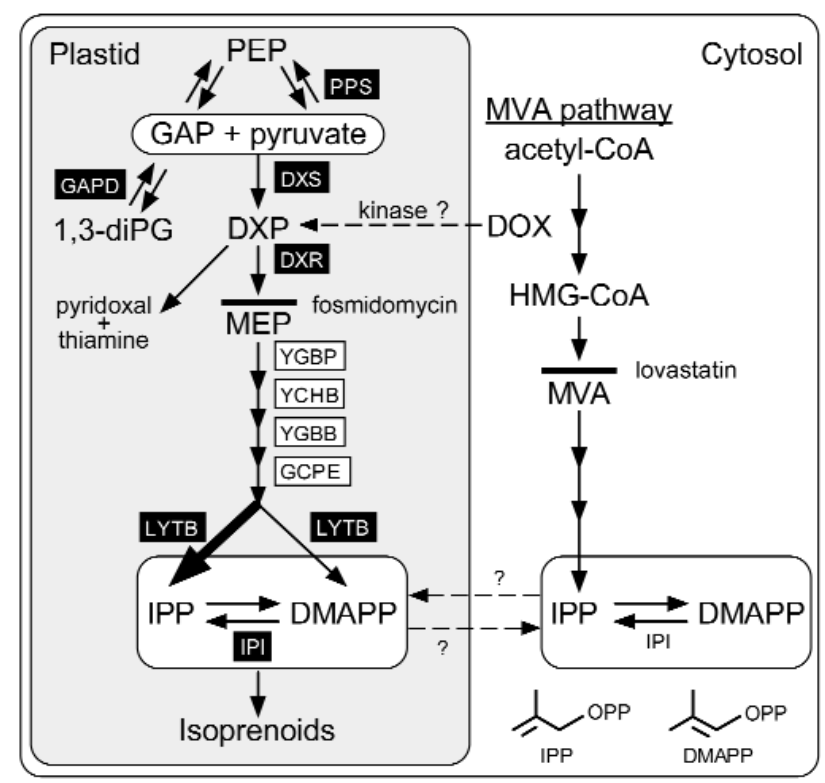

Fig. 1 Distinct isoprenoid pathways exist in plastids and cytosol of plant cells. Enzymes that may limit flux through the MEP pathway (the evidence is largely from studies of the bacterium E. coli) are in white text in black boxes. Abbreviations: diPG, diphosphoglyceraldehyde; GAP, glyceraldehyde-3-phosphate; GAPD, glyceraldehyde-3phosphate dehydrogenase; HMG, hydroxymethylglutaryl; MEP, methylerythritol-5-phosphate; PEP, phosphoenolpyruvate. For other abbreviations, see text. 
pathway inhibitor lovastatin does not [23]. The Arabidopsis CLA1 mutant (a "knockout" of 1 of 3 Dxs genes in Arabidopsis) exhibits a bleached phenotype that can be rescued by the application of DOX (it is thought that this compound is phosphorylated in the plastid to form 1-deoxy-D-xylulose-5-phosphate (DXP; see Fig. 1) [24,25].

Is the supply of substrates (i.e., IPP and DMAPP) a limiting factor in carotenoid synthesis in plants? If so, which enzymatic reactions of the MEP pathway are most restrictive of pathway flux? Perhaps one of the first indications that substrate supply might limit plastid isoprenoid synthesis came from analyses of transgenic tomato plants that had been engineered to overexpress PSY, the initial enzyme of the carotenoid pathway (see Fig. 2), from a constitutive promoter [26]. Such tomato plants were found to be stunted in their growth, arguably due to insufficient synthesis of another class of isoprenoid compounds, the gibberellins, the pathway for which also resides in plastids and apparently competes with carotenoid synthesis for a limited pool of substrate (Fig. 2).

Most of what is known regarding "bottlenecks" of the MEP pathway has come from analyses of carotenoid-accumulating strains of E. coli. Introduction of foreign genes or simply increasing the copy number of the endogenous genes for either IPI [9,27-29] or DXS [29-31] will substantially increase the yield of carotenoids in E. coli. Increases in carotenoid content have also been achieved by increasing expression of the endogenous Dxr gene (but only when Dxs gene expression was also increased) [32] and by coordinating expression of the endogenous phosphoenolpyruvate synthase (PPS) gene with the intracellular metabolic state [33]. Introduction of a plant cDNA encoding the GapA subunit of glyceraldehyde-3-phosphate dehydrogenase (GAPD) will also increase carotenoid accumulation in E. coli [F. X. Cunningham, Jr. and E. Gantt; unpublished data], but only for cultures grown on solid media (carotenoid content of liquid cultures was reduced). The effects of GAPD and PPS on carotenoid accumulation are consistent with the suggestion [33] that availability of glyceraldehyde-3-phosphate (GAP) limits flux through the MEP pathway in E. coli.

Plant cDNAs and cyanobacterial genes encoding LYTB, an enzyme that catalyzes the terminal, branching step of the MEP pathway (Fig. 1) [17,29], also significantly improve carotenoid yield in

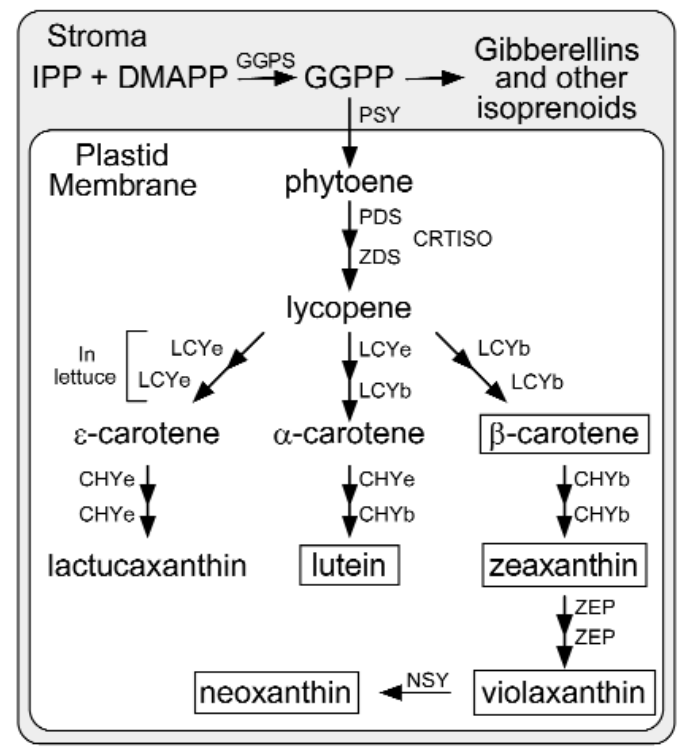

Fig. 2 Pathway of carotenoid synthesis in plant plastids. Carotenoids common in plants are boxed. Abbreviations: DMAPP, dimethylallyl diphosphate; GGPP, geranylgeranyl diphosphate; IPP, isopentenyl diphosphate. Also see Table 1. 
E. coli [29]. Increasing the copy number of the endogenous E. coli LytB gene, however, has no effect on carotenoid accumulation, and the plant $L y t B$ does not further increase carotenoid yield in cultures wherein the Ipi copy number has also been increased [29]. It would appear, therefore, that the plant and cyanobacterial LYTB produce IPP and DMAPP in a proportion more suitable to the needs of carotenoid synthesis (i.e., 3 IPP to 1 DMAPP) than does the E. coli enzyme.

Information as to MEP pathway bottlenecks in plant plastids is limited. Overexpression of a gene encoding DXS, the first enzyme of the pathway, was reported to increase the amounts of several isoprenoids (including a small but significant increase in leaf carotenoids) in transgenic Arabidopsis [34]. Also, injection of 1-deoxy-D-xylulose (DOX; see Fig. 2) was found to accelerate lycopene accumulation in tomato fruits [35], and overexpression of a gene encoding DXR in mint greatly increased the content of certain valuable isoprenoids that constitute the "essential oil" in this plant [36]. In the negative, and in contrast to the positive influence of IPI on carotenoid yield of E. coli, the overexpression of an Ipi from yeast in the cyanobacterium Synechocystis PCC6803 did not increase the carotenoid content [37].

\section{BRANCHING OF THE PATHWAY: THE CYCLIZATION REACTIONS}

The carotenoid pathway branches at the cyclization reactions to produce carotenoids with either two $\beta$-rings (e.g., $\beta$-carotene and derivatives thereof) or one $\varepsilon$ - and one $\beta$-ring (e.g., $\alpha$-carotene and lutein; Fig. 2). The absence of a branch leading to carotenoids with two $\varepsilon$-rings in most plants (lettuce is a rare exception) is explained by the inability of the lycopene $\varepsilon$-cyclase enzyme (LCYe) to add two $\varepsilon$-rings to the symmetrical substrate lycopene, whereas the related $\beta$-cyclase enzyme (LCYb) readily adds two $\beta$-rings [38]. The hypothesis that the relative activities of LCYb and LCYe might serve to apportion flux into the two pathway branches [38,39] has recently received some direct experimental support. Transgenic Arabidopsis plants overexpressing the gene for LCYe accumulate nearly twofold the lutein found in the wild type [6], a substantial alteration since lutein is already the predominant carotenoid in chloroplasts of wild-type Arabidopsis.

LCYe and LCYb are distantly related, with amino acid sequence identities of ca. $35 \%$ overall for a pairwise comparison of the Arabidopsis enzymes. What constrains LCYe to add a single $\varepsilon$-ring to lycopene while LCYb can add two $\beta$-rings? What structural features determine whether a $\beta$ - or $\varepsilon$-ring results? Some progress in answering these questions has recently been made. An amino acid sequence region involved in determining the number of $\varepsilon$-rings introduced by LCYe was mapped by construction and analysis of chimera comprised of different portions of the Arabidopsis LCYe and a romaine lettuce LCYe with the unusual ability to add two $\varepsilon$-rings to lycopene [40]. Site-directed mutagenesis in this region revealed that the identity of a single amino acid residue can determine the number of rings introduced by LCYe. The ability to add two rings may well depend on the formation of enzyme homodimers [40].

Attempts to map sequence determinants of ring type by construction and analysis of chimeric cyclases have met with less success. Chimera of Arabidopsis LCYe and LCYb have not, for the most part, yielded active enzymes. Despite a relatively low overall sequence similarity, plant LCYb and LCYe do share a few well-conserved amino acid sequence motifs (not shown). The spacing between two of the most well-conserved of these shared motifs (MVFMDYRD and PTFLYAMP for LCYe vs. MLFMDWRD and PTFLYAMP for LCYb of Arabidopsis) differs by three amino acids for the known plant LCYe and LCYb. A chimeric $\varepsilon-\beta-\varepsilon$ cyclase was constructed such that the amino acids of the poorly conserved "spacer" region between the two Arabidopsis LCYe motifs were replaced by those of the corresponding spacer region in the Arabidopsis LCYb (the mutant YTNEKVRSLEAEY288300KHLDSYPELKERNSKI). The chimera thereby obtained was found to convert lycopene into several different products, with absorption spectra indicative of the presence of both $\beta$ - and $\varepsilon$-rings [F. X. Cunningham, Jr. and E. Gantt; unpublished data]. The occurrence of both ring types in the mixture of products is consistent with the proposal of Britton [41] that $\beta-, \varepsilon^{-}$, and $\gamma$-rings are formed via a 


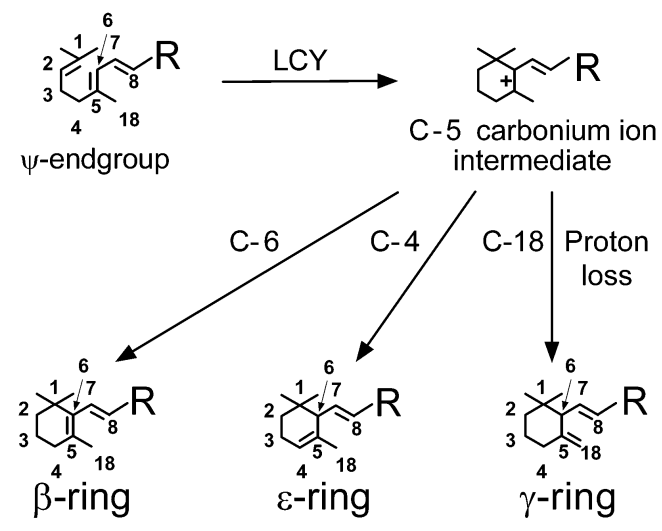

Fig. 3 Formation of $\beta-, \varepsilon$-, and $\gamma$-rings from the linear $\psi$-end group is thought to proceed via a common carbonium ion intermediate with the resulting ring type determined by the particular carbon that provides the leaving proton [41].

common carbonium ion intermediate (Fig. 3) with the resulting ring type determined by the carbon (C-4, C-6, or C-18) that provides the leaving proton. It appears that spacing of the two well-conserved sequence motifs of LCYe and LCYb affects the choice of the leaving proton.

\section{CURRENT AND FUTURE APPROACHES}

The regulatory mechanisms employed by plants to maintain and adjust the amounts and composition of the carotenoid pigments in chloroplasts and chromoplasts are beginning to be revealed. Control of pathway flux may be mediated, in part, by changes in the expression of genes encoding enzymes of the pathway, particularly those for PSY and the cyclases, and by the availability of substrates produced via the MEP pathway. These relatively coarse controls, however, are but facets of a precise and sophisticated system of regulation, many aspects of which are post-translational in nature. What current and future experimental approaches are likely to augment our understanding of the control of carotenoid biosynthesis in plants?

Much of what has been learned about the carotenoid pathway in the last decade has come from the selection and analysis of mutants, primarily of Arabidopsis and tomato. From Arabidopsis mutants we have come to know of the involvement of quinones in the desaturation reactions (the $p d s 1$ mutant) [42], the necessity for an alternative oxidase (PTOX) in non-green plastids (immutans) [43,44], and the need for a carotene isomerase (CRTISO; mutant $c c r 2$ ) [45]. From tomato mutants, we have learned of a fruit-specific PSY (yellow flesh) [46], a linkage between carotenogenesis and light signal transduction (high pigment) [47], the need for an alternative oxidase (ghost) [48], the existence of a second LCYb that also serves as the NSY (high-beta and old-gold crimson) [49], and the need for a carotene isomerase (tangerine) [50]. A number of other Arabidopsis and tomato carotenoid mutants are currently under study, as are some interesting mutants of cauliflower (Or) [51] and carrot $(r p)$ [52].

A related approach that may soon yield new insights into the control of carotenoid biosynthesis in plants makes use of natural variation in the pigmentation of non-green tissues of related species or of different varieties of the same species. High resolution mapping of "quantitative trait loci" (QTLs) that account for differences in carotenoid content may soon lead to the identification of individual genes or clusters of genes that influence carotenoid accumulation. Several studies have already demonstrated the feasibility of this approach in pepper [53] and tomato [54,55 and others], but no genes affecting carotenoid accumulation have yet been identified in this way. 
Another promising experimental approach, one not yet exploited for the study of carotenoid biosynthesis, is "activation tagging", wherein a strong, constitutive promoter is randomly inserted in the genome. An activation-tagged Arabidopsis mutant with intense purple pigmentation due to an accumulation of anthocyanins enabled Borevitz et al. [56], to identify a transcription factor that is involved in regulating the biosynthesis of these pigments.

Global analyses of gene expression using microarrays also may soon provide clues and generate hypotheses as to control mechanisms for carotenoid biosynthesis. The first such foray in this area is a comparison of expression profiles in xanthophyll biosynthesis mutants of Arabidopsis (L. Tian and D. Dellapenna, Abstract \#636, Plant Biology 2001, Providence, RI).

Insights into the regulation of carotenoid biosynthesis also will likely come from efforts now underway to engineer carotenoid metabolism in plants. Some puzzling and unexpected consequences have already been reported. Constitutive expression of a bacterial phytoene desaturase enzyme (CrtI) in transgenic tobacco plants led to an increase in carotenoids with two $\beta$-rings at the expense of those (e.g., lutein) with one $\beta$ - and one $\varepsilon$-ring [57]. Expression of $C R T I$ in tomato fruits produced an increase in $\beta$-carotene but a severe reduction in total carotenoid content [58], whereas overexpression of LCYb led to increases in both $\beta$-carotene and total carotenoids [60]. Most intriguing is a report [61] that fruits of tomato plants disabled in the fruit-specific PSY-1 retain the ability to synthesize phytoene via an active PSY-2 (the enzyme predominant in green tissues) but do not do so. This observation and others, together with several reports of a soluble "phytoene synthase complex" that contains PSY, GGPS, and IPI, [62-64], suggest a metabolic "channeling" of carotenoid pathway intermediates [61]. Enzyme complexes containing the later, membrane-associated enzymes of the pathway (PDS and beyond; see Figs. 2 and 4) have long been thought to exist [41,59]. Much could be learned by going "back to the future" and employing a classical biochemical approach, along with modern tools and methods, to isolate and characterize these membrane-associated carotenoid enzyme complexes.

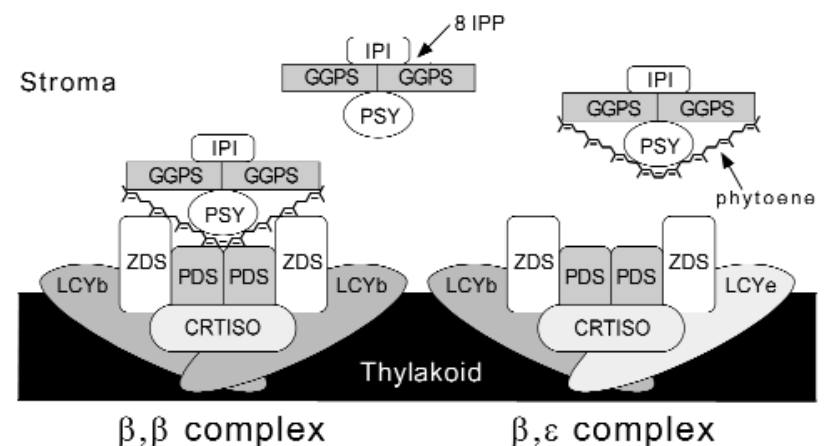

Fig. 4 Hypothetical carotenoid enzyme complexes in plant plastids. After [59]. For abbreviations, see Table 1.

\section{ACKNOWLEDGMENTS}

Supported by grants from NSF (MCB 9631257) and DOE (DE-FG02-98ER20302), with additional support from Monsanto, Quest, and Zeneca.

\section{REFERENCES}

1. A. J. Young. In Carotenoids in Photosynthesis, A. J. Young and G. Britton (Eds.), pp. 16-71, Chapman and Hall, London (1993).

2. A. J. Young. In Carotenoids in Photosynthesis, A. J. Young and G. Britton (Eds.), pp. 161-205, Chapman and Hall, London (1993). 
3. D. DellaPenna. In The Photochemistry of Carotenoids, H. A. Frank, A. J. Young, G. Britton, R. J. Cogdell (Eds.), pp. 21-37, Kluwer Academic Publishers, The Netherlands (1999).

4. B. J. Pogson, K. A. McDonald, M. Truong, G. Britton, D. DellaPenna. Plant Cell 8, 627-1639 (1996).

5. B. J. Pogson, K. K. Niyogi, O. Bjorkman, D. DellaPenna. Proc. Natl. Acad. Sci. USA 95, 13324-13329 (1998).

6. B. J. Pogson and H. M. Rissler. Philos. Trans. R. Soc. London, Ser. B 355, 1395-1403 (2000).

7. H. M. Rissler and B. J. Pogson. Photosyn. Res. 67, 127-137 (2001).

8. P. D. Fraser, S. Romer, C. A. Shipton, P. B. Mills, J. W. Kiano, N. Misawa, R. G. Drake, W. Schuch, P. M. Bramley. Proc. Natl. Acad. Sci. USA 99, 1092-1097 (2002).

9. Z. Sun, E. Gantt, F. X. Cunningham, Jr. J. Biol. Chem. 271, 24349-24352 (1996).

10. L. Tian and D. DellaPenna. Plant Mol. Biol. 47, 379-388 (2001).

11. S. Al-Babili, P. Hugueney, M. Schledz, R. Welsch, H. Frohnmeyer, O. Laule, P. Beyer. FEBS Lett. 485, 168-172 (2000).

12. F. Bouvier, A. D'harlingue, R. A. Backhaus, M. H. Kumagai, B. Camara. Eur. J. Biochem. 267, 6346-6352 (2000).

13. G. Ronen, L. Carmel-Goren, D. Zamir, J. Hirschberg. Proc. Natl. Acad. Sci. USA 97, 11102-11107 (2000).

14. K. Okada, T. Saito, T. Nakagawa, M. Kawamukai, Y. Kamiya. Plant Physiol. 122, 1045-1056 (2000).

15. T. J. Bach, A. Boronat, N. Campos, A. Ferrer, K. U. Vollack. Crit. Rev. Biochem. Mol. Biol. 34, 107-122 (1999).

16. M. Rohmer. Nat. Prod. Rep. 16, 565-574 (1999).

17. F. Rohdich, S. Hecht, K. Gartner, P. Adam, C. Krieger, S. Amslinger, D. Arigoni, A. Bacher, W. Eisenreich. Proc. Natl. Acad. Sci. USA 99, 1158-1163 (2002).

18. M. Rodríguez-Conceptión, N. Campos, L. Maria Lois, C. Maldonado, J. F. Hoeffler, C. Grosdemange-Billiard, M. Rohmer, A. Boronat. FEBS Lett. 473, 328-332 (2000).

19. P. D. Fraser, W. Schuch, P. M. Bramley. Planta 211, 361-369 (2000).

20. S. L. Spurgeon, N. Sathyamoorthy, J. W. Porter. Arch. Biochem. Biophys. 230, 446-454 (1984).

21. H. K. Lichtenthaler. Ann. Rev. Plant Physiol. Plant Mol. Biol. 50, 47-65 (1999).

22. J. G. Zeidler, J. Schwender, C. Mòller, J. Wiesner, C. Weidemeyer, E. Beck, H. Jomaa, H. K. Lichtenthaler. Z. Naturforsch. 53c, 980-998 (1998).

23. M. Rodríguez-Conceptión and W. Gruissem. Plant Physiol. 119, 41-48 (1999).

24. N. Araki, K. Kusumi, K. Masamoto, Y. Niwa, K. Iba. Physiologia Plant. 108, 19-24 (2000).

25. J. M. Estevez, A. Cantero, C. Romero, H. Kawaide, L. F. Jimenez, T. Kuzuyama, H. Seto, Y. Kamiya, P. Leon. Plant Physiol. 124, 95-104 (2000).

26. R. G. Fray, A. Wallace, P. D. Fraser, D. Valero, P. Hedden, P. M. Bramley, D. Grierson. Plant J. 8, 693-701 (1995).

27. S. Kajiwara, P. D. Fraser, K. Kondo, N. Misawa. Biochem. J. 324, 421-426 (1997).

28. F. X. Cunningham, Jr. and E. Gantt. Plant Cell Physiol. 41, 119-123 (2000).

29. F. X. Cunningham, Jr., T. Lafond, E. Gantt. J. Bacteriol. 182, 5841-5848 (2000).

30. M. Harker and P. M. Bramley. FEBS Lett. 448, 115-119 (1999).

31. P. D. Matthews and E. T. Wurtzel. Appl. Microbiol. Biotechnol. 53, 396-400 (2000).

32. S. W. Kim and J. D. Keasling. Biotechnol. Bioeng. 72, 408-415 (2001).

33. W. R. Farmer and J. C. Liao. Biotechnol. Prog. 17, 57-61 (2001).

34. J. M. Estevez, A. Cantero, A. Reindi, S. Reichler, P. Leon. J. Biol. Chem. 276, 22901-22909 (2001).

35. L. M. Lois, M. Rodríguez-Conceptión, F. Gallego, N. Campos, A. Boronat. Plant J. 22, 503-513 (2000).

36. S. S. Mahmoud and R. B. Croteau. Proc. Natl. Acad. Sci. USA 98, 8915-8920 (2001). 
37. D. Lagarde, L. Beuf, W. Vermaas. Appl. Environ. Microbiol. 66, 64-72 (2000).

38. F. X. Cunningham, Jr., B. Pogson, Z. Sun, K. A. McDonald, D. DellaPenna, E. Gantt. Plant Cell 8, 1613-1626 (1996).

39. I. Pecker, R. Gabbay, F. X. Cunningham, Jr., J. Hirschberg. Plant Mol. Biol. 30, 807-819 (1996).

40. F. X. Cunningham, Jr. and E. Gantt. Proc. Natl. Acad. Sci. USA 98, 2905-2910 (2001).

41. G. Britton. Physiol. Veg. 20, 735-755 (1982).

42. S. R. Norris, T. R. Barrette, D. DellaPenna. Plant Cell 7, 2139-2149 (1995).

43. P. Carol, D. Stevenson, C. Bisanz, J. Breitenbach, G. Sandmann, R. Mache, G. Coupland, M. Kuntz. Plant J. 11, 57-68 (1999).

44. D. Wu, D. A. Wright, C. Wetzel, D. F. Voytas, S. Rodermel. Plant Cell 11, 43-55 (1999).

45. H. Park, S. S. Kreunen, A. Cuttris, D. DellaPenna, B. J. Pogson. Plant Cell 14, 321-332 (2002).

46. R. G. Fray and D. Grierson. Plant Mol. Biol. 22, 589-602 (1993).

47. A. C. Mustilli, F. Fenzi, R. Ciliento, F. Alfano, C. Bowler. Plant Cell 11, 145-157 (1999).

48. E. M. Josse, A. J. Simkin, J. Gaffé, A. M. Labouré, M. Kuntz, P. Carol. Plant Physiol. 123, 1427-1436 (2000).

49. G. Ronen, L. Carmel-Goren, D. Zamir, J. Hirschberg. Proc. Natl. Acad. Sci. USA 97, 11102-11107 (2000).

50. T. Isaacson, G. Ronen, D. Zamir, J. Hirschberg. Plant Cell 14, 333-342 (2002).

51. L. Li, D. J. Paolillo, M. V. Parthasarathy, E. M. DiMuzio, D. F. Garvin. Plant J. 26, 59-67 (2001).

52. W. Y. L. Poon and I. L. Goldman. J. Am. Soc. Hort. Sci. 127, 284-289 (2002).

53. T. A. Thorup, B. Tanyolac, K. D. Livingstone, S. Popovsky, I. Paran, M. Jahn. Proc. Natl. Acad. Sci. USA 97, 11192-11197 (2000).

54. D. Bernacchi, T. Beck-Bunn, Y. Eshed, J. Lopez, V. Petiard, J. Uhlig, D. Zamir, S. Tanksley. Theor. Appl. Genet. 97, 381-397 (1998).

55. V. Saliba-Colombani, M. Causse, D. Langlois, J. Philouze, M. Buret. Theor. Appl. Genet. 102, 259-272 (2001).

56. J. O. Borevitz, Y. Xia, J. Blount, R. A. Dixon, C. Lamb. Plant Cell 12, 2383-2394 (2000).

57. N. Misawa, K. Masamoto, T. Horit, T. Ohtani, P. Böger, G. Sandmann. Plant J. 6, 481-489 (1994).

58. P. D. Fraser, S. Romer, J. W. Kiano, C. A. Shipton, P. B. Mills, R. Drake, W. Schuch, P. M. Bramley. J. Sci. Food Agr. 81, 822-827 (2001).

59. F. X. Cunningham, Jr. and E. Gantt. Ann. Rev. Plant Physiol. Plant Mol. Biol. 49, 557-583 (1998).

60. C. Rosati, R. Aquilani, S. Dharmapuri, P. Pallara, C. Marusic, R. Tavazza, F. Bouvier, B. Camara, G. Giuliano. Plant J. 24, 413-419 (2000).

61. P. D. Fraser, J. W. Kiano, M. R. Truesdale, W. Schuch, P. M. Bramley. Plant Mol. Biol. 40, 687-698 (1999).

62. J. W. Porter and S. L. Spurgeon. Pure Appl. Chem. 51, 609-622 (1979).

63. B. Camara. Methods Enzymol. 214, 352-365 (1993).

64. M. Lützow and P. Beyer. Biochim. Biophys. Acta 959, 118-126 (1988). 